\title{
On the Use of Antipodal Optimal Dimensionality Sampling Scheme on the Sphere for Recovering Intra-voxel Fibre Structure in Diffusion MRI
}

\author{
Alice P. Bates, Zubair Khalid and Rodney A. Kennedy \\ Research School of Engineering, The Australian National University, Canberra, ACT \\ 2601, Australia \\ \{alice.bates, zubair.khalid, rodney.kennedy\}@anu.edu.au
}

\begin{abstract}
In diffusion magnetic resonance imaging (dMRI), the diffusion signal can be reconstructed from measurements collected on single or multiple spheres in $\boldsymbol{q}$-space using a spherical harmonic expansion. The number of measurements that can be acquired is severely limited and should be as small as possible. Previous sampling schemes have focused on using antipodal symmetry to reduce the number of samples and uniform sampling to achieve rotationally invariant reconstruction accuracy, but do not allow for an accurate or computationally efficient spherical harmonic transform (SHT). The recently proposed antipodal optimal dimensionality sampling scheme on the sphere requires the minimum number of samples, equal to the number of degrees of freedom for the representation of the antipodal symmetric band-limited diffusion signal in the spherical harmonic domain. In addition, it allows for the accurate and efficient computation of the SHT. In this work, we evaluate the use of this recently proposed scheme for the reconstruction of the diffusion signal and subsequent intra-voxel fibre structure estimation in dMRI. We show, through numerical experiments, that the use of this sampling scheme allows accurate and computationally efficient reconstruction of the diffusion signal, and improved estimation of intra-voxel fibre structure, in comparison to the antipodal electrostatic repulsion and spherical code sampling schemes with the same number of samples. We also demonstrate that it achieves rotationally invariant reconstruction accuracy to the same extent as the other two sampling schemes.
\end{abstract}

\section{Introduction}

Diffusion magnetic resonance imaging (dMRI) uses the intra-voxel diffusion characteristics of water molecules to determine the structure and connectivity of white matter in the brain. Diffusion signal measurements are collected on a single sphere or multiple spheres in $\boldsymbol{q}$-space (known as $\boldsymbol{q}$-shells) $[8,10]$. The reconstruction of the diffusion signal on a sphere from these measurements is carried out by expanding the signal in terms of spherical harmonics $[11,14]$. By choosing a sufficiently large band-limit in the spherical harmonic degree, $L$, the diffusion signal can be represented in terms of a finite number of coefficients 
in the spectral domain, enabled by spherical harmonic transform (SHT) [16]. Various techniques proposed in the literature for estimating the intra-voxel fibre structure in dMRI use the diffusion signal spherical harmonic coefficients, such as Q-ball imaging in constant solid angle $\left(\mathrm{QBI}_{\mathrm{CSA}}\right)[1,12]$.

In order to facilitate accurate and fast estimation of fibre structure, a sampling scheme must support accurate and efficient computation of the SHT. It is also important that the scheme require as few measurements as possible in order to reduce scan times $[2,19]$. Furthermore as fibre populations may assume any orientation within a voxel, the accuracy of the reconstruction of the diffusion signal should not change significantly if the diffusion signal (or sampling scheme) is rotated $[8,7]$. As dMRI is an inherently noisy imaging technique, reconstruction should also be robust to noise [9].

Novel Sampling Scheme Many sampling schemes used in dMRI focus on uniform sampling on the sphere to achieve rotationally invariant reconstruction accuracy and antipodally symmetric sampling grids to reduce the number of samples, but do not consider accurate and efficient computation of the SHT $[7,8,15]$. Recently, the antipodal optimal dimensionality sampling scheme on the sphere [3] has been proposed for the reconstruction of antipodal symmetric signals. This scheme enables a SHT which is more computationally efficient than the other sampling schemes that use the least squares (LS) method of SHT computation, as we show later in the paper. It also requires the minimum number of samples, given by the degrees of freedom required to represent the antipodal symmetric signal in the spectral domain, for accurate computation of SHT of the signal.

The widely used antipodal electrostatic repulsion sampling scheme (ESR) [15] can be used with the minimum number of samples, however it does not allow accurate reconstruction of the diffusion signal with this number of points, as we later demonstrate in the paper. The sampling scheme [7] generalises the ESR scheme to multiple $\boldsymbol{q}$-shells and reduces to the ESR scheme for a single $\boldsymbol{q}$-shell. The ESR scheme is also extended to 3D $\boldsymbol{q}$-space sampling in [17] and a generalised metric is defined. For single-shell $\boldsymbol{q}$-space sampling, the energy measure used in ESR is suitable [17]. The scheme [8] is another scheme with a uniformly and antipodally symmetric distributing of samples on the sphere, it generalises the spherical code (SC) formulation (minimum angular distance between samples) to multiple shells and can be formed for any number of samples.

We note that the sampling scheme [6] uses spherical design to enable the accurate computation of the SHT, and has a uniform and antipodally symmetric arrangement of samples, however it requires more than the minimum number of samples. The equiangular sampling scheme proposed in [10] has an accurate and efficient SHT but requires approximately four times the minimum number of samples.

Contributions In this work, we evaluate the antipodal optimal dimensionality sampling scheme for estimating the intra-voxel fibre structure in dMRI. We 
address whether the sampling scheme: A) enables the accurate reconstruction of the diffusion signal on the sphere and consequently improves estimation of fibre structure within each voxel, B) has a reconstruction accuracy that does not vary significantly with rotation and $\mathrm{C}$ ) is computationally efficient.

In order to answer these questions, we evaluate the antipodal optimal dimensionality sampling scheme against other single $\boldsymbol{q}$-shell sampling schemes that can be used with the minimum number of samples; we analyse the reconstruction of the diffusion signal and the intra-voxel fibre structure estimation using $\mathrm{QBI}_{\mathrm{CSA}}$ from measurements of the diffusion signal taken over the antipodal optimal dimensionality, ESR and SC sampling schemes. We demonstrate that the acquisition of measurements over the antipodal optimal dimensionality sampling scheme allows accurate and computationally efficient reconstruction of the diffusion signal, and better estimation of intra-voxel structure in dMRI.

\section{Materials and Methods}

\subsection{Diffusion Signal on Sphere}

Let the diffusion weighted signal at a fixed $\boldsymbol{q}$-space radius (or fixed diffusion weighting, $b)$ be denoted by $S(\theta, \phi ; b)$, where the angles co-latitude $\theta \in[0, \pi]$ and longitude $\phi \in[0,2 \pi)$ parameterise a point $\boldsymbol{u}(\theta, \phi)=(\sin \theta \cos \phi, \sin \theta \sin \phi, \cos \theta)^{\prime}$ on the sphere $\mathbb{S}^{2}$.

The spherical harmonic functions (or spherical harmonics for short), denoted by $Y_{\ell}^{m}(\theta, \phi)$ and defined for integer degree $\ell \geq 0$ and integer order $|m| \leq \ell$, form a complete basis for the space of signals defined on the sphere. Since $S(\theta, \phi ; b)$ is antipodal symmetric, with $S(\theta, \phi ; b)=S(\pi-\theta, \phi+\pi ; b)$ and $Y_{\ell}^{m}(\theta, \phi)=$ $Y_{\ell}^{m}(\pi-\theta, \pi+\phi)$ for even $\ell$ and $Y_{\ell}^{m}(\theta, \phi)=-Y_{\ell}^{m}(\pi-\theta, \pi+\phi)$ for odd $\ell$, the expansion of $S(\theta, \phi ; b)$ in the spherical harmonic basis only includes even degree spherical harmonics, that is,

$$
S(\theta, \phi ; b)=\sum_{\substack{\ell=0 \\ \ell \text { even }}}^{L-1} \sum_{m=-\ell}^{\ell}(S)_{\ell}^{m}(b) Y_{\ell}^{m}(\theta, \phi), \quad L \text { odd },
$$

where $L$ represents the band-limit that depends on the $b$-value $[10,19]$, and $(S)_{\ell}^{m}(b)$ denotes the spherical harmonic coefficient of degree $\ell$ and order $m$, which is calculated using the SHT, given by

$$
(S)_{\ell}^{m}(b) \triangleq \int_{\mathbb{S}^{2}} S(\theta, \phi ; b) \overline{Y_{\ell}^{m}(\theta, \phi)} \sin \theta d \theta d \phi
$$

The spherical harmonic coefficients $(S)_{\ell}^{m}(b)$ form the spectral domain representation of $S(\theta, \phi ; b)$. In practise, $(2)$ has to be calculated numerically; there exist sampling schemes, such as [3], that enable algorithms for accurately calculating the SHT (see [18] for a comprehensive review). For other schemes, such as [8] and $[15],(S)_{\ell}^{m}(b)$ can also be calculated using LS where (1) is written as a system of linear equations. 
The reconstruction of the signal $S(\theta, \phi ; b)$ from its spherical harmonic coefficients, as given in (1), is referred to as the inverse SHT. The diffusion signal is assumed to be band-limited at degree $L$ such that $(S)_{\ell}^{m}(b)=0$ for $\ell \geq L$; if this assumption does not hold, there is a truncation error.

Diffusion-Weighted Data Synthesis We use the commonly used Gaussian mixture model $[2,9]$ to obtain a diffusion weighted dataset. The diffusion weighted signal model for a voxel is given by

$$
S(\theta, \phi ; b)=S_{0} \sum_{k=1}^{M} f_{k} e^{-b \boldsymbol{u}(\theta, \phi)^{T} \boldsymbol{D}_{k} \boldsymbol{u}(\theta, \phi)},
$$

where $S_{0}$ is the baseline image at $b=0, M$ is the number of fibres, the volume fractions $f_{k}$ of each fibre population are normalized to ensure that $\sum_{k=1}^{M} f_{k}=1$ and $\boldsymbol{D}_{k}$ is the diffusion tensor for the $k$ th fibre in the voxel. Each fibre's tensor is computed from a rotated version of a tensor, $\boldsymbol{D}=\operatorname{diag}\left(\lambda_{1}, \lambda_{2}, \lambda_{3}\right)$, with $\boldsymbol{D}_{k}=$ $\boldsymbol{R}_{k}^{T} \boldsymbol{D} \boldsymbol{R}_{k}$, where $\lambda_{1}$ is the diffusivity along the main axis of a fibre while $\lambda_{2}$ and $\lambda_{3}$ are the diffusivities in the plane perpendicular to it, and $\boldsymbol{R}_{k}$ is the rotation matrix that rotates the $k$ th fibre to the direction of the $k$ th fibre population.

In the numerical experiments where the effect of noise is considered, we add Rician noise to the diffusion weighted signal as [13]

$$
S(\theta, \phi ; b)_{n}=\sqrt{\left(S(\theta, \phi ; b)+\eta_{1}\right)^{2}+\eta_{2}^{2}},
$$

with $\eta_{1}, \eta_{2} \sim \mathcal{N}\left(0, \sigma^{2}\right)$ and $\sigma=S_{0} / \mathrm{SNR}$. The signal-to-noise ratio (SNR) controls the level of noise on the baseline image, assumed to be $S_{0}=1$ [9].

\subsection{Antipodal Optimal Dimensionality Sampling Scheme}

The antipodal optimal dimensionality sampling scheme [3], which we denote by $\mathfrak{S}_{O}\left(N_{\mathrm{O}}\right)$ where $N_{\mathrm{O}}=L(L+1) / 2$, has an iso-latitude sampling grid with $L$ iso-latitude rings placed in antipodal pairs at $\left[0, \ldots, \pi-\theta_{L-3}, \theta_{L-3}, \pi-\right.$ $\left.\theta_{L-1}, \theta_{L-1}\right], L$ odd and equiangular sampling along longitude with the points placed so that the samples in ring $\theta_{n}$ are antipodal to those in ring $\theta_{n-1}$ (Fig. 1 shows $\mathfrak{S}_{O}(28)$ which has $L=7$ rings). The antipodal nature of $\mathfrak{S}_{O}\left(N_{\mathrm{O}}\right)$ means that measurements only need to be taken over the rings $\theta_{n}$ for $n=0,2, \ldots, L-1$; the value of the diffusion signal over the remaining points can be determined using the antipodal symmetry of the diffusion signal.

The number of measurements required by this scheme is $N_{\mathrm{O}}$, which is the minimum number of samples attainable by any sampling scheme as there are $N_{\mathrm{O}}$ degrees of freedom required to represent the antipodal band-limited signal in the spectral domain (as can be seen from Eq.(1)). More details of $\mathfrak{S}_{O}\left(N_{\mathrm{O}}\right)$, including the precise location of samples, can be found in [3]. The design of $\mathfrak{S}_{O}\left(N_{\mathrm{O}}\right)$ enables a SHT (described in [3]) which is accurate and efficient, unlike other schemes that focus on uniform sampling of the sphere which use LS. 


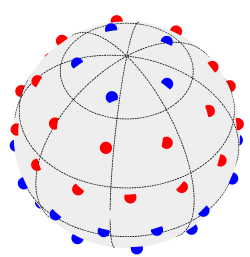

(a)

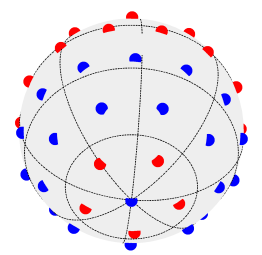

(b)

Fig. 1: The antipodal optimal dimensionality sampling scheme for $L=7, \mathfrak{S}_{O}(28)$ a) north and b) south pole view. Points where measurements are taken are shown in blue and points where antipodal symmetry is used to evaluate $S(\theta, \phi ; b)$ are shown in red.

\section{Diffusion Signal Reconstruction}

In this section, we analyse the reconstruction of the diffusion signal from its samples taken over $\mathfrak{S}_{O}\left(N_{\mathrm{O}}\right)$. We obtain a diffusion weighted dataset using the diffusion signal model (3), with $\lambda_{1}=1.7 \times 10^{-3} \mathrm{~mm}^{2} / \mathrm{s}$ and $\lambda_{2}=\lambda_{3}=$ $0.3 \times 10^{-3} \mathrm{~mm}^{2} / \mathrm{s}$ which are values typically observed in the human brain [5] .

\subsection{Evaluation of Band-limit of Diffusion Signal}

It has been demonstrated that $\mathfrak{S}_{O}\left(N_{\mathrm{O}}\right)$ allows accurate computation of the SHT of any band-limited antipodal symmetric signal on the sphere in [3]; we therefore evaluate whether the assumption that the diffusion signal is band-limited holds. In order to study the band-limit of the diffusion signal, we define the per-degree energy spectrum $P(b, \ell)$ of the diffusion signal (3) as

$$
P(b, \ell) \triangleq \sum_{m=-\ell}^{\ell}\left|(S)_{\ell}^{m}(b)\right|^{2},
$$

which is plotted for $1000 \leq b \leq 8000 \mathrm{~s} / \mathrm{mm}^{2}$ and $0 \leq \ell \leq 45$ in Fig. 2a, where it can be observed that the energy spectrum $P(b, \ell)$ decreases gradually with the increase in spherical harmonic degree $\ell$.

The threshold lines on the surface plot in Fig. 2a for which $P(b, \ell)$ drops below $10^{-15}$ (black) and $10^{-6}$ (grey) can guide us in choosing the band-limit of the diffusion signal. For example, $L=21$ and $L=11$ for $b=3000 \mathrm{~s} / \mathrm{mm}^{2}$ as indicated by black and grey line, respectively. For $L=21$, we require $N_{\mathrm{O}}=231$ samples, which may be too large as it is common for around 60 samples to be taken at $b=3000 \mathrm{~s} / \mathrm{mm}^{2}[9]$. For $L=11$, we need $N_{\mathrm{O}}=66$ samples. Using a smaller $L$ (larger threshold) means less samples are required but will result in a larger truncation error, as we demonstrate in the next section.

The approximately linear relationship between $b$ and $L$ is indicated by dashed lines in Fig. 2a. Fig. 2a shows $P(b, \ell)$ for a synthetic diffusion signal obtained from (3) with $M=2$ fibres and a crossing angle of $25^{\circ}$, however we observed insignificant variation in $P(b, \ell)$ for different $M$ and fibre orientations. 


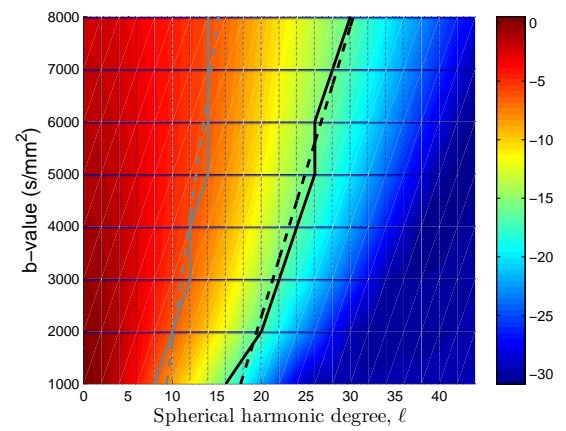

(a)

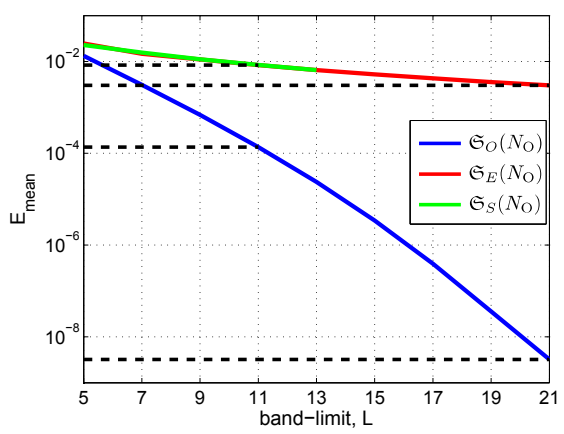

(b)

Fig. 2: (a) Per-degree energy spectrum $P(b, \ell)(5)$, is plotted as $\log _{10} P(b, \ell)$ for $0 \leq$ $\ell<45, \ell$ odd and $1000 \leq b \leq 8000 \mathrm{~s} / \mathrm{mm}^{2}$. Black and grey lines indicate where $P(b, \ell)$ drops below $10^{-15}$ and $10^{-6}$ respectively, and dashed lines show linear relationship between $b$ and $L$. (b) Mean reconstruction error $E_{\text {mean }}$ for $5 \leq L \leq 21$ for $\mathfrak{S}_{O}\left(N_{\mathrm{O}}\right)$, $\mathfrak{S}_{E}\left(N_{\mathrm{O}}\right)$ and $\mathfrak{S}_{S}\left(N_{\mathrm{O}}\right)$.

\subsection{Reconstruction Accuracy}

In order to evaluate whether $\mathfrak{S}_{O}\left(N_{\mathrm{O}}\right)$ allows for the accurate reconstruction of $S(\theta, \phi ; b)$, we compare it with the ESR scheme [15] composed of $N_{\mathrm{O}}$ samples, denoted by $\mathfrak{S}_{E}\left(N_{\mathrm{O}}\right)$ and the SC scheme [8] composed of $N_{\mathrm{O}}$ samples, denoted by $\mathfrak{S}_{S}\left(N_{\mathrm{O}}\right)^{1}$. In order to compute the SHT of a signal from its samples taken over $\mathfrak{S}_{E}\left(N_{\mathrm{O}}\right)$ and $\mathfrak{S}_{S}\left(N_{\mathrm{O}}\right)$, regularised LS is used with regularisation parameter $\lambda=0.006$ (used in $[12,20]$ ) to improve the condition number of the matrix involved the computation of the SHT. Due to space constraints, here and in the rest of the paper we only show results for $b=3000 \mathrm{~s} / \mathrm{mm}^{2}$, which is commonly used to obtain $S(\theta, \phi)$ measurements [9].

We conduct the following experiment to determine the reconstruction accuracy. For a given $b$-value, synthetic measurements of the diffusion signal, using (3), are first obtained over the sampling grid $\mathfrak{S}_{O}\left(N_{\mathrm{O}}\right)$ or $\mathfrak{S}_{E}\left(N_{\mathrm{O}}\right)$ or $\mathfrak{S}_{S}\left(N_{\mathrm{O}}\right)$. The spherical harmonic coefficients $(S)_{\ell}^{m}(b)$ are then calculated using the SHT proposed in [3] or the regularised LS method [12]. Finally, the spherical harmonic coefficients $(S)_{\ell}^{m}(b)$ are used to reconstruct the diffusion signal over a high resolution uniform grid (consisting of 2562 points which are the vertices of a 4th-order icosahedron). We analyse the mean reconstruction error, $E_{\text {mean }} \triangleq \operatorname{mean}\left(\left|S_{A}(\theta, \phi ; b)-S_{r}(\theta, \phi ; b)\right|\right)$, between the reconstructed and analytical value of the diffusion signal calculated over the 2562 points.

\footnotetext{
1 The best known solutions of the SC problem [8] are available at http://neilsloane.com/grass/dim3/ for up to 100 antipodal pairs, hence we are only able to show results obtained using SC for $L<15\left(N_{\mathrm{O}}=91\right)$ in this paper.
} 


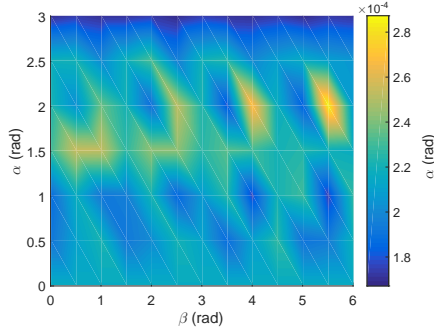

(a)

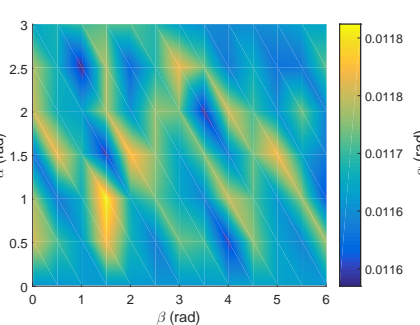

(b)

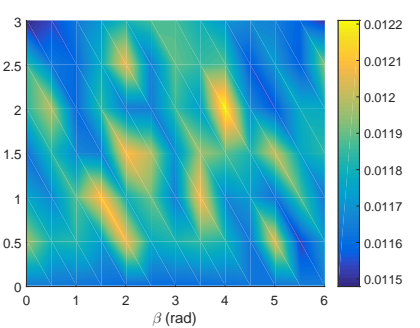

(c)

Fig. 3: Mean reconstruction error $E_{\text {mean }}$ varies insignificantly for different fibre orientations, given by $\alpha \in[0, \pi]$ and $\beta \in(0,2 \pi]$, for (a) $\mathfrak{S}_{O}(66)$, (b) $\mathfrak{S}_{E}(66)$ and (c) $\mathfrak{S}_{S}(66)$.

The mean reconstruction error $E_{\text {mean }}$ for band-limits $5 \leq L \leq 21$ is shown in Fig. $2 \mathrm{~b}$, where it is evident that taking measurements over $\mathfrak{S}_{O}\left(N_{\mathrm{O}}\right)$, in comparison to $\mathfrak{S}_{E}\left(N_{\mathrm{O}}\right)$ and $\mathfrak{S}_{S}\left(N_{\mathrm{O}}\right)$, enables significantly more accurate reconstruction of the diffusion signal. For example at $L=21\left(N_{O}=231\right)$, the mean error $E_{\text {mean }}$ is on the order of $10^{-9}$ and $10^{-4}$ for $\mathfrak{S}_{O}\left(N_{\mathrm{O}}\right)$ and $\mathfrak{S}_{E}\left(N_{\mathrm{O}}\right)$ respectively, while at $L=11\left(N_{\mathrm{O}}=66\right)$, it is on the order of $10^{-4}$ for $\mathfrak{S}_{O}\left(N_{\mathrm{O}}\right)$, and $10^{-2}$ for $\mathfrak{S}_{E}\left(N_{\mathrm{O}}\right)$ and $\mathfrak{S}_{S}\left(N_{\mathrm{O}}\right)$. Fig. $2 \mathrm{~b}$ shows $E_{\text {mean }}$ for a synthetic diffusion signal obtained from (3) with $M=2$ fibres and a crossing angle of $25^{\circ}$ as in Section 3.1, however we again observed insignificant variation in $E_{\text {mean }}$ for different $M$ and fibre orientations. In summary, the use of the sampling scheme $\mathfrak{S}_{O}\left(N_{\mathrm{O}}\right)$ greatly reduces the diffusion signal reconstruction error compared with $\mathfrak{S}_{E}\left(N_{\mathrm{O}}\right)$ and $\mathfrak{S}_{S}\left(N_{\mathrm{O}}\right)$.

Rotational Invariance $\mathfrak{S}_{E}\left(N_{\mathrm{O}}\right)$ and $\mathfrak{S}_{S}\left(N_{\mathrm{O}}\right)$ focus on uniform sampling of the sphere to ensure rotationally invariant reconstruction accuracy $[15,8]$, that is the accuracy of reconstruction does not significantly vary if the diffusion signal (or sampling scheme is rotated) [7]. $\mathfrak{S}_{O}\left(N_{\mathrm{O}}\right)$ is not uniform by design, however, it does not have dense sampling on any region of the sphere. For all three sampling schemes $\mathfrak{S}_{O}\left(N_{\mathrm{O}}\right), \mathfrak{S}_{E}\left(N_{\mathrm{O}}\right)$ and $\mathfrak{S}_{S}\left(N_{\mathrm{O}}\right)$, we analyse the rotational invariance of the reconstruction accuracy; we change the orientation of a fibre centered at $z$-axis by rotating the fibre by $\beta \in[0, \pi]$ around $y$-axis and then by $\alpha \in$ $[0,2 \pi]$ around $x$-axis, and compute the mean reconstruction error $E_{\text {mean }}$ for different orientations/rotations. In Fig. 3(a), (b) and (c) it can be observed that the mean error $E_{\text {mean }}$ does not change significantly for any of the schemes (the reconstruction error remains on the order if $10^{-4}$ for $\mathfrak{S}_{O}\left(N_{\mathrm{O}}\right)$, and on the order of $10^{-2}$ for $\mathfrak{S}_{E}\left(N_{\mathrm{O}}\right)$ and $\mathfrak{S}_{S}\left(N_{\mathrm{O}}\right)$ for all rotations), showing that all schemes enable rotationally invariant reconstruction accuracy to the same extent.

\subsection{Computation Time}

We investigate the computational complexity of the SHT of $\mathfrak{S}_{O}\left(N_{\mathrm{O}}\right)[3] \mathrm{com}-$ pared to the LS method of SHT computation employed by $\mathfrak{S}_{E}\left(N_{\mathrm{O}}\right)$ and $\mathfrak{S}_{S}\left(N_{\mathrm{O}}\right)$ 


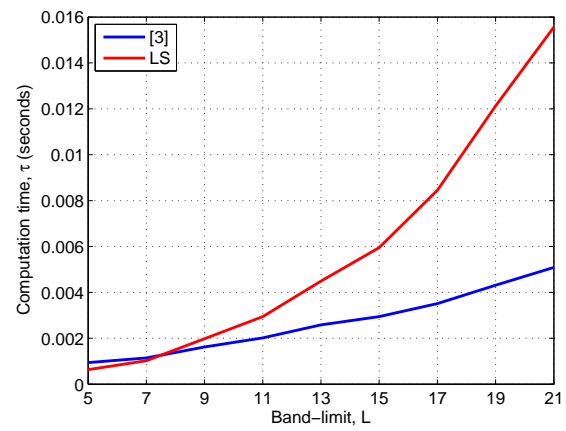

Fig. 4: The computation time $\tau$ in seconds for the SHT in [3] and for LS to compute the spherical harmonic coefficients of the diffusion signal for $5 \leq L \leq 21$.

by measuring the time it takes for both methods to calculate the spherical harmonic coefficients of the diffusion signal for band-limits $5 \leq L \leq 21$. It can be seen in Fig. 4 that for $L>7$, [3] allows for faster computation of the SHT and that the time taken by the LS method increases much faster with $L$; this is due to the computational complexity of the LS being $O\left(L^{6}\right)$ while the SHT proposed in [3] has asymptotic complexity $O\left(L^{4}\right)$. [3]. Hence, $\mathfrak{S}_{O}\left(N_{\mathrm{O}}\right)$ allows for a SHT which is significantly more efficient compared with the LS method of SHT used by $\mathfrak{S}_{E}\left(N_{\mathrm{O}}\right)$ and $\mathfrak{S}_{S}\left(N_{\mathrm{O}}\right)$.

\section{Application: Intra-voxel Fibre Structure Estimation Using $\mathrm{QBI}_{\mathrm{CSA}}$}

In this section, we show that the acquisition of diffusion signal measurements over $\mathfrak{S}_{O}\left(N_{\mathrm{O}}\right)$ allows accurate estimation of the intra-voxel structure in dMRI. In our analysis, we use $\mathrm{QBI}_{\mathrm{CSA}}[1]^{2}$, one of the intra-voxel structure estimation techniques that uses the spherical harmonic coefficients of the diffusion signal, that has been compared in the 2012 High Angular Resolution Diffusion (HARDI) Reconstruction Challenge [12]. We use the structured field testing data set $^{3}$, that consists of 1280 voxels, where each voxel is constructed using the diffusion signal model given in (3) (see [9] for the values of parameters). To compare the performance of $\mathfrak{S}_{O}\left(N_{\mathrm{O}}\right)$ with $\mathfrak{S}_{E}\left(N_{\mathrm{O}}\right)$ and $\mathfrak{S}_{S}\left(N_{\mathrm{O}}\right)$ we use the following two metrics: success rate (SR) defined as the percentage of voxels in which the correct number of fibre populations are detected and mean average angular error per voxel, denoted by mean $(\bar{\theta})$, defined as the average error between the estimated fibre directions and the true ones in each voxel, averaged over all voxels.

\footnotetext{
${ }^{2}$ The orientation distribution function (ODF) peaks are extracted using finite differences over a 724 point grid mesh as in [12].

3 available at http://hardi.epfl.ch/static/events/2012_ISBI/
} 


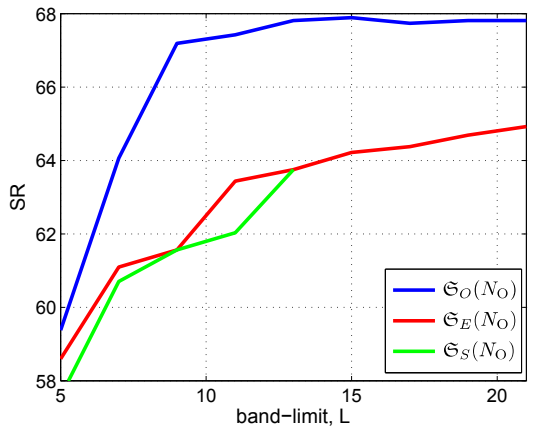

(a)

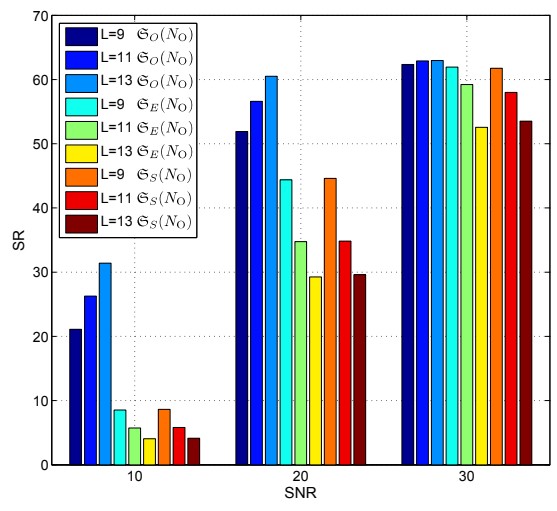

(c)

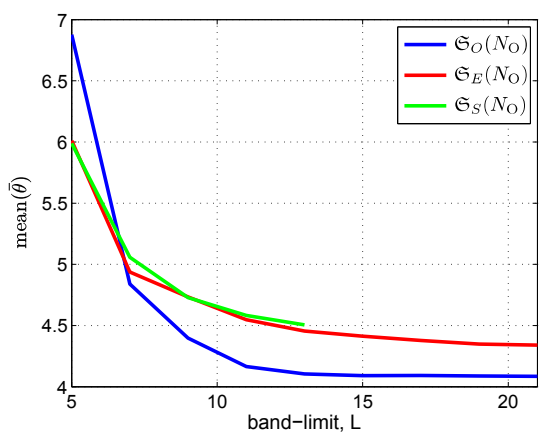

(b)

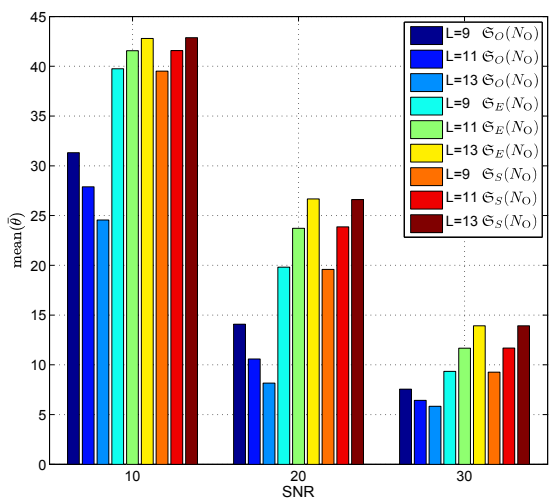

(d)

Fig. 5: Fibre reconstruction metrics, a) SR and b) mean $(\bar{\theta})$, obtained by sampling over $\mathfrak{S}_{O}\left(N_{\mathrm{O}}\right), \mathfrak{S}_{E}\left(N_{\mathrm{O}}\right)$ and $\mathfrak{S}_{S}\left(N_{\mathrm{O}}\right)$ for $5 \leq L \leq 21$ in the absence of noise. (c) SR and (d) $\operatorname{mean}(\bar{\theta})$ for $\mathrm{L}=9,11$ and 13 , and $\mathrm{SNR}=10,20$ and 30, and sampling schemes $\mathfrak{S}_{O}\left(N_{\mathrm{O}}\right), \mathfrak{S}_{E}\left(N_{\mathrm{O}}\right)$ and $\mathfrak{S}_{S}\left(N_{\mathrm{O}}\right)$.

Noise Free We have plotted the performance metrics, SR and mean $(\bar{\theta})$, Fig. 5a and $\mathrm{b}$ for noise free measurements obtained using $\mathfrak{S}_{O}\left(N_{\mathrm{O}}\right), \mathfrak{S}_{E}\left(N_{\mathrm{O}}\right)$ and $\mathfrak{S}_{S}\left(N_{\mathrm{O}}\right)$. It is evident that the higher diffusion signal reconstruction accuracy of $\mathfrak{S}_{O}\left(N_{\mathrm{O}}\right)$, compared with $\mathfrak{S}_{E}\left(N_{\mathrm{O}}\right)$ and $\mathfrak{S}_{S}\left(N_{\mathrm{O}}\right)$, results in a higher SR and lower mean $(\bar{\theta})$. In the absence of noise, $\mathfrak{S}_{O}\left(N_{\mathrm{O}}\right)$ therefore enables more accurate intra-voxel fibre structure estimation compared with $\mathfrak{S}_{E}\left(N_{\mathrm{O}}\right)$ and $\mathfrak{S}_{S}\left(N_{\mathrm{O}}\right)$.

With Noise We use 2012 HARDI Challenge Data with SNR = 10, 20 and 30 (4) to evaluate the intra-voxel fibre structure estimation performance of $\mathfrak{S}_{O}\left(N_{\mathrm{O}}\right)$ used with $\mathrm{QBI}_{\mathrm{CSA}}$ in the presence of noise. The regularisation parameter in LS used for $\mathfrak{S}_{E}\left(N_{\mathrm{O}}\right)$ and $\mathfrak{S}_{S}\left(N_{\mathrm{O}}\right)$ filters the noise [12]. For $\mathfrak{S}_{O}\left(N_{\mathrm{O}}\right)$, we use a 
Gaussian kernel given by $e^{-\ell(\ell+1) t}[4]$, with parameter $t=0.032$ (empirically chosen to maximise SR), to low pass filter the noisy signal. More sophisticated filtering techniques that take into account the noise statistics is future work.

Fig. 5c and Fig. 5d show the SR and mean $(\bar{\theta})$ respectively for different SNR averaged over 10 realisations of the noise for $\mathfrak{S}_{O}\left(N_{\mathrm{O}}\right), \mathfrak{S}_{E}\left(N_{\mathrm{O}}\right)$ and $\mathfrak{S}_{S}\left(N_{\mathrm{O}}\right)$ for $L=9,11,13\left(N_{\mathrm{O}}=45,66,91\right)$, which are typical numbers for single-shell sampling). $\mathfrak{S}_{O}\left(N_{\mathrm{O}}\right)$ has a higher SR and lower mean $(\bar{\theta})$ than $\mathfrak{S}_{E}\left(N_{\mathrm{O}}\right)$ and $\mathfrak{S}_{S}\left(N_{\mathrm{O}}\right)$ for all SNR, demonstrating that the estimation of fibre structure from noisy measurements taken over $\mathfrak{S}_{O}\left(N_{\mathrm{O}}\right)$ is more accurate.

\section{Conclusions}

In this work, we have evaluated the antipodal optimal dimensionality sampling scheme on the sphere for the reconstruction of the diffusion signal and subsequent intra-voxel structure estimation in dMRI. Unlike other schemes in the literature, this scheme allows for the accurate and efficient computation of the SHT with the minimum number of measurements. The antipodal optimal dimensionality scheme achieved a greater diffusion signal reconstruction and intra-voxel fibre structure estimation accuracy in the absence and presence of noise, in comparison to the antipodal electrostatic repulsion and spherical code sampling schemes when the minimum number of samples were used. It has also been shown that all three schemes give rotationally invariant reconstruction accuracy to the same extent. Extension of the work to multiple $\boldsymbol{q}$-shell sampling and the analysis of the scheme with real data is being carried out.

\section{References}

1. Aganj, I., Lenglet, C., Sapiro, G., Yacoub, E., Ugurbil, K., Harel, N.: Reconstruction of the orientation distribution function in single- and multiple-shell Q-ball imaging within constant solid angle. Magn. Reson. Med. 64(2), 554-566 (Aug 2010)

2. Assemlal, H.E., Tschumperlé, D., Brun, L.: Evaluation of q-space sampling strategies for the diffusion magnetic resonance imaging. In: Med. Image Comput. Comput. Assist. Interv., MICCAI'2009, vol. 12, pp. 406-414. London, UK (2009)

3. Bates, A.P., Khalid, Z., Kennedy, R.A.: An optimal dimensionality sampling scheme on the sphere for antipodal signals in diffusion magnetic resonance imaging. Arxiv preprint physics.med-ph/1502.07099 presented at ICASSP'2015 (2015)

4. Bülow, T.: Multiscale image processing on the sphere. In: Proceedings of the 24th DAGM Symposium on Pattern Recognition. pp. 609-617. London, UK (2002)

5. Canales-Rodríguez, E.J., Melie-García, L., Iturria-Medina, Y.: Mathematical description of q-space in spherical coordinates: Exact Q-ball imaging. Magnetic Resonance in Medicine 61(6), 1350-1367 (2009)

6. Caruyer, E., Deriche, R.: A computational framework for experimental design in diffusion MRI. In: MICCAI Workshop on Computational Diffusion MRI, CDMRI'2012. Nice, France (Oct 2012)

7. Caruyer, E., Lenglet, C., Sapiro, G., Deriche, R.: Design of multishell sampling schemes with uniform coverage in diffusion MRI. Magn. Reson. Med. 69(6), 15341540 (Jun 2013) 
8. Cheng, J., Shen, D., Yap, P.T.: Designing single- and multiple-shell sampling schemes for diffusion MRI using spherical code. In: Med. Image Comput. Comput. Assist. Interv., MICCAI'2014, vol. 8675, pp. 281-288. Boston, Massachusetts (2014)

9. Daducci, A., Canales-Rodrìguez, E.J., Descoteaux, M., Garyfallidis, E., Gur, Y., Lin, Y.C., Mani, M., Merlet, S., Paquette, M., Ramirez-Manzanares, A., Reisert, M., Reis Rodrigues, P., Sepehrband, F., Caruyer, E., Choupan, J., Deriche, R., Jacob, M., Menegaz, G., Prčkovska, V., Rivera, M., Wiaux, Y., Thiran, J.P.: Quantitative comparison of reconstruction methods for intra-voxel fiber recovery from diffusion MRI. IEEE Trans. Med. Imag. 33(2), 384-399 (Feb 2014)

10. Daducci, A., McEwen, J.D., Ville, D.V.D., Thiran, J.P., Wiaux, Y.: Harmonic analysis of spherical sampling in diffusion MRI. In: Proc. 19th Ann. Meet. Int. Soc. Magn. Reson. Med. (Jun 2011)

11. Descoteaux, M., Angelino, E., Fitzgibbons, S., Deriche, R.: Regularized, fast, and robust analytical Q-ball imaging. Magn. Reson. Med. 58(3), 497-510 (Sep 2007)

12. Descoteaux, M., Boré, A.: Testing classical single-shell HARDI techniques. In: Proceedings of the 2012 ISBI Diffusion MRI reconstruction contest/workshop. p. 5 . Barcelona, Spain (2012)

13. Gudbjartsson, H., Patz, S.: The rician distribution of noisy MRI data. Magn. Reson. Med. 34(6), 910-914 (Dec 1995)

14. Hess, C.P., Mukherjee, P., Han, E.T., Xu, D., Vigneron, D.B.: Q-ball reconstruction of multimodal fiber orientations using the spherical harmonic basis. Magn. Reson. Med. 56(1), 104-117 (Jul 2006)

15. Jones, D.K., Horsfield, M.A., Simmons, A.: Optimal strategies for measuring diffusion in anisotropic systems by magnetic resonance imaging. Magn. Reson. Med. 42(3), 515-525 (Sep 1999)

16. Kennedy, R.A., Sadeghi, P.: Hilbert Space Methods in Signal Processing. Cambridge University Press, Cambridge, UK (Mar 2013)

17. Knutsson, H., Westin, C.F.: Tensor metrics and charged containers for 3d qspace sample distribution. In: Med. Image Comput. Comput. Assist. Interv., MICCAI'2013. vol. 16, pp. 679-86. Nagoya, Japan (Sep 2013)

18. McEwen, J.D., Wiaux, Y.: A novel sampling theorem on the sphere. IEEE Trans. Signal Process. 59(12), 5876-5887 (Dec 2011)

19. Tournier, J.D., Calamante, F., Connelly, A.: Determination of the appropriate $\mathrm{b}$ value and number of gradient directions for high-angular-resolution diffusionweighted imaging. NMR Biomed. 26(12), 1775-1786 (Dec 2013)

20. Wilkins, B., Lee, N., Rajagopalan, V., Law, M., Leporé, N.: Effect of data acquisition and analysis method on fiber orientation estimation in diffusion MRI. In: Computational Diffusion MRI and Brain Connectivity, pp. 13-24 (2014) 